\title{
Mechanisms of Melatonin-Induced Protection in the Brain of Late Gestation Fetal Sheep in Response to Hypoxia
}

\author{
Tamara Yawno Margie Castillo-Melendez Graham Jenkin Euan M. Wallace \\ David W. Walker Suzanne L. Miller \\ The Ritchie Centre, Monash Institute of Medical Research, and Department of Obstetrics and Gynaecology, \\ Monash University, Clayton, Vic., Australia
}

\section{Key Words}

Melatonin · Neuroprotection · Fetal brain - Sheep ·

Umbilical cord occlusion

\begin{abstract}
Melatonin has diverse physiological actions in addition to its well-recognized maintenance roles in circadian and seasonal timing. In particular, melatonin may have a direct protective action on the developing fetal brain. We examined the cellular processes by which melatonin provides protection following an acute late gestation hypoxic insult. 15 fetal sheep at 126 days' gestation were instrumented with a brachial artery catheter and a silastic cuff around the umbilical cord. At $~ 130$ days' gestation, the cuff was inflated for $10 \mathrm{~min}$ in 10 fetuses, causing complete umbilical cord occlusion (UCO). 5 UCO fetuses received intravenous melatonin maternally for $2 \mathrm{~h}$, before and after UCO (UCO + melatonin). The remaining 5 fetuses had no UCO performed (sham-operated controls). At $48 \mathrm{~h}$ after UCO, the fetal brain was collected from each animal. Compared to controls, UCO caused significant hypoxia, hypercapnia and acidosis in UCO and UCO + melatonin fetuses. In the UCO-alone animals there were significant increases in pyknotic cell death, in the hippocampus (>7-fold) and the cerebellum (3-fold). Maternal melato-
\end{abstract}

nin administration ameliorated cellular pyknosis in UCO fetuses. UCO was also associated with astrogliosis, increased albumin uptake, activated microglia and lipid peroxidation. Melatonin prevented these effects. There were no significant differences in the number of brain macrophages or microglia between any of the groups. Following acute severe hypoxia in the late gestation fetus, melatonin reduces neuronal lipid peroxidation and prevents loss of blood-brain barrier integrity and astrogliosis. These are likely key mechanisms underlying the neuroprotective actions of melatonin in the fetal brain.

Copyright $\odot 2013$ S. Karger AG, Basel

\section{Introduction}

Perinatal hypoxia-ischemia is thought to be one of the principal causes of neonatal encephalopathy [1] and subsequent neurodevelopmental impairment and cerebral palsy [2]. Brief but severe fetal hypoxia-ischemia can be induced experimentally in pregnant animals by umbilical cord occlusion (UCO), resulting in perinatal brain injury similar to that in human babies, and therefore assisting with our knowledge of the mechanisms and susceptibility of the brain to injury [3-5]. While the pattern of brain

\section{KARGER}

Fax +4161306 1234

E-Mail karger@karger.ch

www.karger.com
(C) 2013 S. Karger AG, Basel

0378-5866/12/0346-0543\$38.00/0

Accessible online at:

www.karger.com/dne
Dr. Suzanne Miller

The Ritchie Centre

Monash Institute of Medical Research

27-31 Wright St, Clayton, VIC 3168 (Australia)

E-Mail suzie.miller@monash.edu 
injury depends on a number of factors such as gestational age $[6,7]$, and severity and length of the asphyxia [4, 6-9], it is generally agreed that white matter brain injury predominates in the preterm brain, reflecting the sensitivity of oligodendrocyte precursors to injury [10-12], whereas region-specific neuronal loss in the hippocampus, basal ganglia and cerebellum is more common in late gestation $[4,6,7,12,13]$.

The sequence of events following a severe hypoxic episode includes not only a primary wave of cell death, which is principally necrotic, but also a secondary phase of delayed and sustained energy failure during which many more cells either die or are committed to a programmed (apoptotic) cell death pathway $[12,14,15]$. Importantly, oxidative stress has a role in both the early and secondary phases of cerebral tissue damage, as shown by the biphasic increase in hydroxyl radical formation that occurs in grey and white matter of the fetal sheep brain following acute in utero hypoxia in late gestation [16]. The fetal brain has a high lipid content, making it vulnerable to the effects of both oxygen and nitrogen free radicals on protein and lipid structure and function and against which endogenous antioxidant enzymes have limited capacity to protect $[17,18]$.

Antioxidant therapy may prevent or ameliorate brain tissue damage following hypoxia [19-22]. In support of this proposal, we have shown that the administration of melatonin prior to an acute hypoxic event in the late gestation sheep fetus abolishes both the primary and secondary increases in brain hydroxyl radical formation and reduces cerebral lipid peroxidation [23]. Others have shown that melatonin reduces oxidative stress and cell damage in the immature fetal sheep brain in response to severe hypoxia [20], and, in fetal and neonatal rats, melatonin improves indices of brain mitochondrial injury and protects against hippocampal cell loss following cerebral ischemia and reperfusion [24-26]. Melatonin also demonstrates anti-inflammatory properties, stimulates placental expression of antioxidant enzymes, increases placental efficiency and enhances umbilical flow in compromised pregnancies [27-29]; all actions which are likely to protect the developing fetus.

This study was undertaken to investigate whether melatonin reduces the cellular responses to injury within the brain following a brief asphyxial episode. We hypothesized that acute fetal hypoxia, as could occur in perinatal hypoxia-ischemia, induces brain oxidative stress and inflammation in the late gestation brain, and melatonin mediates oxidative and inflammatory responses to reduce brain damage.

\section{Experimental Procedures}

\section{Animals}

Fifteen pregnant Merino-Border Leicester ewes of known gestational age, carrying singleton fetuses, were used for this study. The ewes were kept in individual cages with free access to food and water under a $12 \mathrm{~h}$ light/dark cycle (lights on 07:00 h, off 19:00 h). The use of these animals as well as the procedures performed was in accordance with the Code of Practice for the Care and Use of Animals for Scientific Purposes of the National Health and Medical Research Council of Australia, and had received prior approval from the School of Biomedical Sciences, Monash University Standing Committee on Ethics and Animal Experimentation.

\section{Surgical Preparation}

At $126 \pm 2$ days' gestation (term is about 147 days) surgery was performed under $2-3 \%$ halothane (Merial, Parramatta, N.S.W., Australia) general anesthesia for implantation of fetal catheters and an umbilical cuff. Using a midline abdominal incision, the pregnant uterus was identified, opened and the fetal head and right forelimb exteriorized. A sterile polyvinyl catheter $(1.5 \mathrm{~mm}$ outer diameter, $0.8 \mathrm{~mm}$ inner diameter) was inserted into the fetal right brachial artery to allow fetal arterial blood sampling. An inflatable silastic cuff (16HD; In Vivo Medical, Ukiah, Calif., USA) was placed around the umbilical cord which, when inflated with 2-3 $\mathrm{ml}$ of sterilized water, would cause complete cessation of umbilical blood flow. Once instrumented, the fetus was returned to the uterus and the uterus was repaired in two layers. The catheters were exteriorized from the maternal abdomen through a small flank incision. A maternal jugular vein catheter $(2.7 \mathrm{~mm}$ outer diameter, $1.5 \mathrm{~mm}$ inner diameter) was also implanted for melatonin infusion. The ewe and fetus were allowed 3-4 days of recovery before beginning the experiment.

\section{Experimental Design}

At approximately 130 days' gestation, animals were randomly assigned to either a sham UCO (control) group in which the ewe received a vehicle infusion $(n=5), \mathrm{UCO}+$ vehicle group (UCO; $\mathrm{n}$ $=5)$, or UCO + melatonin group $(n=5)$. Melatonin (Sigma-Aldrich, Castle Hill, N.S.W., Australia) was dissolved in absolute ethanol and diluted to $0.08 \mathrm{mg} / \mathrm{ml}$ using sterile physiological saline, so that the concentration of ethanol in the final solution was $1 \%$. At 12:00 h, a 1-mg melatonin bolus in $5 \mathrm{ml}$ saline was administered to the ewe's jugular vein, followed by infusion of $10 \mathrm{ml}$ of $0.2 \mathrm{mg} /$ $\mathrm{ml}$ melatonin over $2 \mathrm{~h}$. Control animals received an equal volume of the vehicle. One hour after the start of melatonin infusion, a 10min complete UCO was performed by inflating the cuff with sterile water. For the control group, the cuff was not inflated. Fetal $(2-\mathrm{ml})$ blood samples were taken before, during and after the start of the occlusion, with $0.5 \mathrm{ml}$ of fetal blood used immediately for measurement of $\mathrm{pH}$, oxygen saturation $\left(\mathrm{O}_{2} \%\right), \mathrm{pO}_{2}$ and $\mathrm{pCO}_{2}$, using an ABL 510 blood gas analyzer (Radiometer, Copenhagen, Denmark). The ewe and fetus were killed $48 \mathrm{~h}$ after the start of UCO or control by an intravenous injection of pentobarbitone sodium (Lethabarb; Virbac Pty Ltd, Peakhurst, N.S.W., Australia) administered to the ewe. The fetal brain was immediately perfused via the carotid artery with 1 liter of $4 \%$ paraformaldehyde (ProSci Tech, Thuringowa, Qld., Australia) in $0.1 \mathrm{M}$ phosphate buffer $(\mathrm{pH}$ 7.4). The brain was then removed from the skull and post-fixed in $4 \%$ paraformaldehyde for $24 \mathrm{~h}$, prior to embedding in paraffin. 
Subsequently, $10 \mu \mathrm{m}$ coronal sections of the fetal brain were cut and stained with hematoxylin and eosin for identification of cellular pyknosis and adjacent sections were used for immunohistochemistry.

\section{Immunohistochemistry}

All sections were placed on SuperFrost Plus glass slides and dewaxed in xylene and rehydrated through a series of ethanol dilutions. Sections were coverslipped using aqueous mounting medium (Dako Australia, Campbellfield, Vic., Australia), after drying at room temperature, for fluorescent sections or using DEPX (Merck, Kilsyth, Vic., Australia) for non-fluorescent sections. For each immunohistochemical protocol, sections containing a specific brain region from all treatment groups were included in a single run to eliminate inter-run variation.

For single-label immunohistochemistry, sections were heated with citric acid buffer for $3 \times 5$ min followed by incubation in $3 \%$ hydrogen peroxide in methanol to remove endogenous peroxidase activity. Sections were then incubated in blocking solution (phosphate-buffered saline, PBS; $0.1 \mathrm{~mol} / \mathrm{l}, \mathrm{pH} 7.4$ ), $0.25 \%$ Triton X-100 and $5 \%$ normal goat serum for $1 \mathrm{~h}$ at room temperature to block non-specific binding, followed by incubation with primary antibody in either anti-glial fibrillary acidic protein (GFAP) antibody to identify mature astrocytes (1:400; Hoffmann-La Roche Ltd, Basel, Switzerland), anti-human/mouse activated caspase-3 antibody to identify apoptosis (1:1,000; R\&D Systems, Minneapolis, Min., USA), 4-hydroxynonenal (4-HNE) rabbit polyclonal antibody to identify lipid peroxidation (1:1,000; Millipore Corp., Billerica, Mass., USA), rabbit anti-sheep albumin to detect blood protein extravasation (1: 1,000; Accurate Chemical \& Scientific Corp., Westbury, N.Y., USA), or rabbit anti-ionized calcium binding adaptor molecule 1 (Iba-1) to identify activated microglia (1:500; Wako Pure Chemical Industries, Ltd, Osaka, Japan) in $0.2 \%$ Tween- 20 for $24 \mathrm{~h}$ at $4^{\circ} \mathrm{C}$. Sections were then incubated in a 1:200 dilution of secondary antibody (biotinylated anti-rabbit or anti-mouse IgG antibody; Vector Laboratories, Burlingame, Calif., USA) for $1 \mathrm{~h}$ at room temperature. Sections were washed in PBS and incubated in horseradish peroxidase (1:200 dilution in PBS for $1 \mathrm{~h}$; Vector Laboratories) and staining was revealed using 3,3'-diaminobenzidine (Pierce Biotechnology, Rockford, Ill., USA). Immunopositive cells were counted under light microscopy (Olympus, Tokyo, Japan). GFAP expression was calculated using Image (version 10.2; National Institutes of Health, Bethesda, Md., USA). Two sections of each brain region per animal were examined, the number of immunopositive cells per region was calculated using the average of four fields of view per section, and the results were averaged across all the animals in each group $(\mathrm{n}=5$ control, $\mathrm{n}=5 \mathrm{UCO}, \mathrm{n}=5 \mathrm{UCO}+$ melatonin).

Double-label immunohistochemistry was carried out on two adjacent sections from 3 control fetuses, 3 UCO and 3 UCO + melatonin fetal brains by first blocking endogenous peroxidases with $0.3 \%$ hydrogen peroxidase in $50 \%$ methanol and then washing sections with sodium borohydride $(10 \mathrm{mg} / \mathrm{ml})$ in $0.1 \mathrm{M}$ PBS to reduce the autofluorescence that can occur with paraffin-embedded sections. These sections were treated with a serum-free protein blocker (Dako) to prevent background staining, and incubated with either monoclonal anti-GFAP (1:400), or anti-NeuN (1:200; Chemicon Int., Temecula, Calif., USA) to identify mature astrocytes or mature neurons, respectively. Sections were subsequently exposed to the appropriate rabbit polyclonal antibody to identify either 4-HNE $(1: 1,000)$, or activated caspase-3 (1:1,000). Immuno-
Table 1. Fetal arterial blood gas parameters

\begin{tabular}{|c|c|c|c|}
\hline & Control & UCO & $\mathrm{UCO}+$ melatonin \\
\hline \multicolumn{4}{|l|}{$\mathrm{SaO}_{2}, \%$} \\
\hline Pre-UCO & $65.4 \pm 3.6$ & $69.4 \pm 5.8$ & $66.7 \pm 4.6$ \\
\hline During UCO & $68.4 \pm 2.2$ & $10.8 \pm 1.8^{*}$ & $11.4 \pm 3.6^{*}$ \\
\hline Post-UCO & $67.5 \pm 3.5$ & $71.2 \pm 4.8$ & $63.1 \pm 6.1$ \\
\hline \multicolumn{4}{|l|}{$\mathrm{P}_{\mathrm{a}} \mathrm{O}_{2}, \mathrm{~mm} \mathrm{Hg}$} \\
\hline Pre-UCO & $22.3 \pm 0.5$ & $22.2 \pm 1.9$ & $21.3 \pm 2.0$ \\
\hline During UCO & $22.4 \pm 0.7$ & $8.5 \pm 2.7^{*}$ & $8.7 \pm 1.8^{\star}$ \\
\hline Post-UCO & $22.2 \pm 0.7$ & $26.6 \pm 2.0$ & $20.9 \pm 2.3$ \\
\hline \multicolumn{4}{|l|}{$\mathrm{P}_{\mathrm{a}} \mathrm{CO}_{2}, \mathrm{~mm} \mathrm{Hg}$} \\
\hline Pre-UCO & $47.1 \pm 1.2$ & $47.9 \pm 1.1$ & $49.8 \pm 1.4$ \\
\hline During UCO & $44.8 \pm 1.7$ & $112.5 \pm 3.0^{*}$ & $103.4 \pm 13.4^{\star}$ \\
\hline Post-UCO & $46.3 \pm 1.5$ & $46.8 \pm 0.4$ & $49.5 \pm 1.7$ \\
\hline \multicolumn{4}{|l|}{$\mathrm{pH}_{\mathrm{a}}$} \\
\hline Pre-UCO & $7.36 \pm 0.01$ & $7.36 \pm 0.01$ & $7.36 \pm 0.01$ \\
\hline During UCO & $7.37 \pm 0.01$ & $6.94 \pm 0.01^{\star}$ & $7.00 \pm 0.06^{\star}$ \\
\hline Post-UCO & $7.36 \pm 0.01$ & $7.29 \pm 0.02^{\star}$ & $7.30 \pm 0.01^{*}$ \\
\hline
\end{tabular}

Values are mean \pm SEM. Three time-points are pre-cord occlusion (pre-UCO) at $-1 \mathrm{~h}$ relative to occlusion, 10 min into cord occlusion (during UCO) and $1 \mathrm{~h}$ after cord occlusion (post-UCO). Significant differences $(\mathrm{p}<0.05)$ are shown as ${ }^{*}$ in control vs. UCO groups, and control vs. UCO + melatonin groups. No differences were detected in UCO vs. UCO + melatonin groups.

reactivity was visualized with Alexa Fluor 594 goat anti-mouse (1: 1,000; Molecular Probes, Eugene, Oreg., USA) and Alexa Fluor 488 goat anti-rabbit (1:1,000; Molecular Probes), and viewed with a fluorescent microscope (Olympus) at $400 \times$ magnification.

\section{Statistical Analysis}

Data are presented as mean \pm SEM. Physiological data over time were analyzed using a two-way repeated-measures analysis of variance with Tukey post hoc analysis. Cell counts were analyzed by one-way ANOVA and Bonferroni adjustment. Statistical significance was set at $\mathrm{p} \leq 5$. Statistical comparisons were carried out using SPSS.

\section{Results}

Prior to cord occlusion, all fetuses had fetal arterial blood gas, oxygen saturation and $\mathrm{pH}$ parameters that were similar between groups and normal for late gestation fetal sheep [4] (table 1). Compared to that observed in control animals, the 10-min UCO caused significant fetal hypoxemia, hypercapnia and acidosis in both UCO and $\mathrm{UCO}+$ melatonin groups (table 1). There were no significant differences in blood gases between the UCO and the $\mathrm{UCO}+$ melatonin fetuses. 


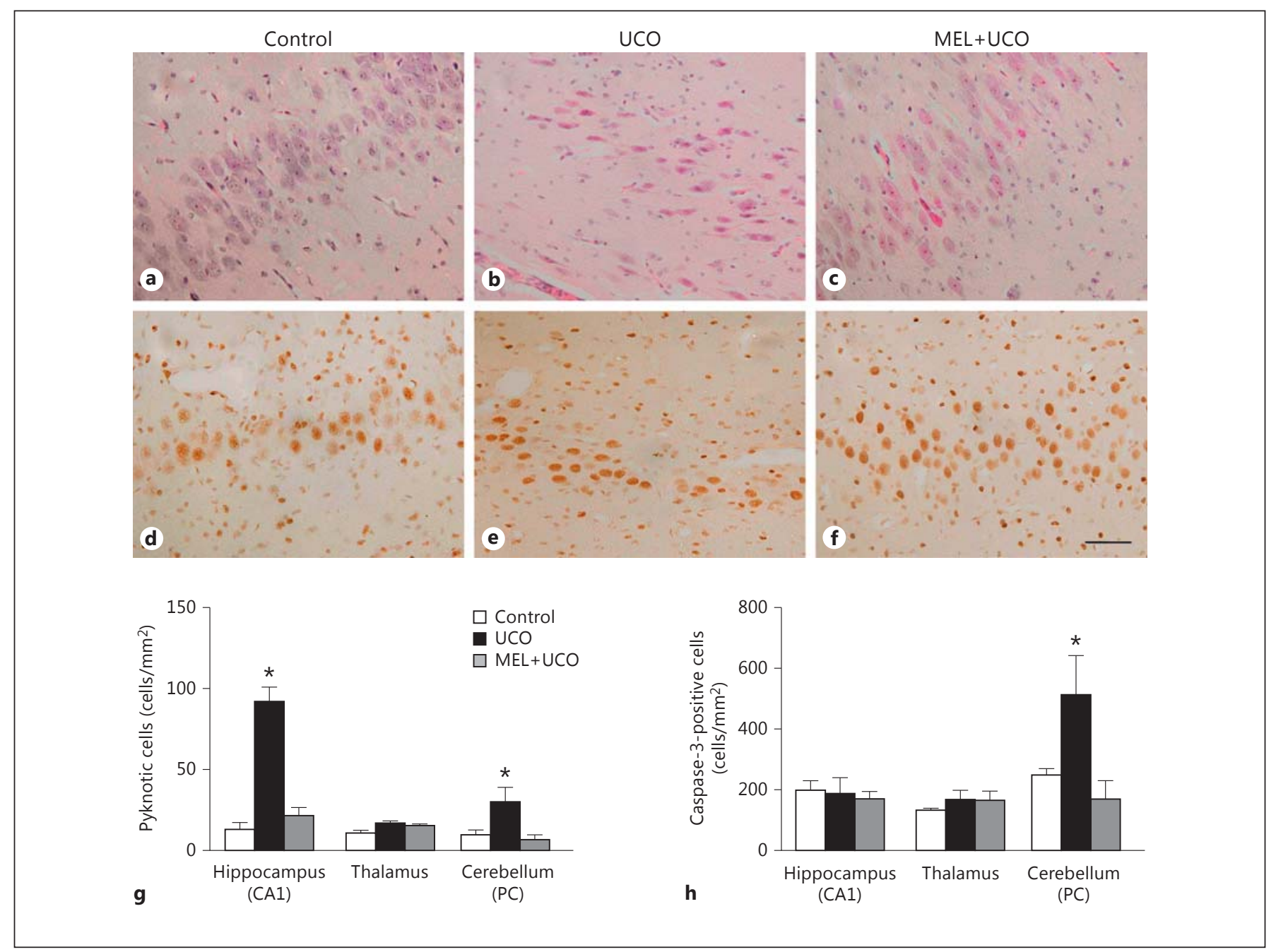

Fig. 1. Photomicrographs showing pyknotic cells (a-c) and activated caspase-3-positive cells (d-f) in the CA1 area of the hippocampus in the fetal brain $48 \mathrm{~h}$ after the sham procedure (a, d), $\mathrm{UCO}(\mathbf{b}, \mathbf{e})$ and UCO + melatonin $(\mathbf{c}, \mathbf{f})$. Scale bar $=50 \mu \mathrm{m}$. The number of pyknotic cells $(\mathbf{g})$ and activated caspase-3-positive cells

Circadian rhythms for circulating fetal melatonin concentrations were observed in all animals over the pretreatment period prior to melatonin administration and UCO. There was no difference in daytime $(9.8 \pm 1.2 \mathrm{pM})$ or nighttime $(45.1 \pm 11.2 \mathrm{pM})$ melatonin concentrations between experimental groups. Maternal administration of melatonin significantly increased fetal arterial plasma melatonin concentration such that after $1 \mathrm{~h}$, fetal levels of melatonin in the UCO + melatonin group were 7,351 $\pm 1,393 \mathrm{pM}$ compared to $13.4 \pm 4.0 \mathrm{pM}$ in the UCO group $(\mathrm{p}<0.001)$. Three hours after cessation of the melatonin infusion (i.e. $4 \mathrm{~h}$ after the cord occlusion) fetal melatonin levels were not significantly different between UCO (h) in the CA1 area of the hippocampus, the thalamus and the Purkinje cell layer (PC) of the cerebellum, in the fetal brain $48 \mathrm{~h}$ after the sham procedure $(\mathrm{n}=5), \mathrm{UCO}(\mathrm{n}=5)$ and $\mathrm{UCO}+$ melatonin $(\mathrm{n}=5)$. Each bar represents the mean $\pm S E M ;{ }^{*} \mathrm{p}<0.05$, control versus UCO.

groups; $15.8 \pm 3.2 \mathrm{pM}$ in $\mathrm{UCO}$ versus $44.8 \pm 12.8 \mathrm{pM}$ in $\mathrm{UCO}+$ melatonin animals; $\mathrm{p}>0.05$.

It has been reported previously that UCO for $10 \mathrm{~min}$ in late gestation fetal sheep results in cell death, particularly in the hippocampus, basal ganglia/thalamus, and cerebellum [4]. Accordingly, we assessed whether melatonin protected against cell death in these regions of the late gestation fetal brain at $48 \mathrm{~h}$ after acute cord occlusion. Pyknotic degenerating neurons were identified using hematoxylin and eosin as shrunken cells with small, densely stained nuclei and eosinophilic cytoplasm. Low numbers of pyknotic cells were observed in the brains of control animals (fig. 1a, g). In UCO animals we observed a 7-fold ( $\mathrm{p}<$ 
0.0001) increase in pyknotic cells within the CA1 of the hippocampus (fig. 1b, g), compared to controls, and similarly, within the Purkinje layer of the cerebellum UCO fetuses showed a 3 -fold $(\mathrm{p}<0.05)$ increase in pyknotic cells versus control fetuses (fig. 1g). Administration of melatonin ameliorated cellular pyknosis in UCO fetuses, such that there was no difference in pyknotic cell numbers in the CA1, thalamus or cerebellum of control and $\mathrm{UCO}+$ melatonin fetuses (fig. 1c, f, g). In addition, caspase-3-positive apoptotic cells were observed in the CA1 region of the hippocampus, thalamus and cerebellum of control, UCO and $\mathrm{UCO}+$ melatonin fetuses. There was a significant increase in the number of caspase-3-positive cells within the Purkinje layer of the cerebellum in UCO fetuses and this effect was mitigated by melatonin administration (fig. $1 \mathrm{~h}$ ). However there was no significant effect of UCO or melatonin treatment on the number of caspase-3-positive cells within the hippocampus or thalamus.

After UCO, there was a marked increase in the level of staining of GFAP-immunopositive astrocytes within the CA1 region of the fetal hippocampus, the thalamus and the Purkinje layer of the cerebellum, compared with control fetal brains (fig. 3a; $\mathrm{p}<0.05$ ). In response to UCO, astrocytes were frequently present and exhibited altered morphology with longer and more numerous processes and larger heavily-stained cell bodies (fig. 2b). These morphological changes were not observed in fetuses in which the ewe had received melatonin (fig. 2c) and UCO + melatonin fetal brains demonstrated decreased GFAP expression compared to UCO alone and were not different to controls (fig. 3a; p < 0.05). As expected, astrocytic processes were found in close association with blood vessels (fig. $2 \mathrm{a}-\mathrm{c}$ ), and these processes also showed increased GFAP immunoreactivity and hypertrophic astrocyte end-feet after UCO (fig. 2b). Plasma albumin was observed in brain parenchyma of UCO fetal brains (fig. 2e), with a significant upregulation of albumin immunoreactivity within hippocampal CA1, thalamus and cerebellar Purkinje cells in UCO versus control brains (fig. $3 \mathrm{~b}$; $\mathrm{p}<$ $0.05)$. Albumin immunoreactivity was observed intracellularly in UCO fetal brains, indicative of cellular sequestration of albumin by cells and most notable within the thalamus and cerebellum (images not shown). Albumin staining was rarely seen in UCO + melatonin fetuses in any brain region (fig. 2f), similar to control fetal brains (fig. 2d) and quantification demonstrated that albumin immunoreactivity was not different in $\mathrm{UCO}+$ melatonin fetuses and controls (fig. 3b). Within the UCO fetal brains, intense albumin staining was notable in the parenchyma adjacent to blood vessels, indicative of increased blood

Neuroprotective Melatonin in the Fetal Brain vessel permeability (fig. 2e) [30]. We quantified the number of albumin-positive vessels and found that UCO induced a significant increase within the thalamus (fig. 3c), compared to control $(\mathrm{p}<0.05)$. The increase in the number of cells and vessels staining positively for albumin following UCO was significantly reduced by prior administration of melatonin to the ewe (fig. 2f, 3b, c).

We assessed the number of Iba-1-positive microglial cells within control, UCO and UCO + melatonin fetal brains. UCO resulted in a significant increase in Iba-1-labeled cells within the cerebellum, compared to control animals $(\mathrm{p}=0.05)$ and this effect was ameliorated in $\mathrm{UCO}+$ melatonin animals (fig. 3c). There was no difference in the number of Iba-1-positive microglia across experimental groups within hippocampal CA1 or thalamus (fig. 3c).

Within the hippocampus, thalamus and cerebellum, no 4-HNE-positive cells were observed in control fetuses, indicative of minimal or no brain lipid peroxidation under normal fetal conditions. UCO significantly increased cellular lipid peroxidation in the hippocampus (fig. $2 \mathrm{k}$, $3 e ; p=0.005$ ) and thalamus (fig. $3 e ; p=0.05$; fig. $5 \mathrm{~h}$ ) and the melatonin treatment in UCO + melatonin fetuses completely abolished this lipid peroxidation response (fig. $2 \mathrm{l}, 3 \mathrm{e} ; \mathrm{p}>0.05$ vs. control). Within the hippocampus we used double-label immunohistochemistry to demonstrate that it was predominantly neurons that were susceptible to lipid peroxidation following UCO, with 70 $80 \%$ of 4 -HNE-positive (lipid peroxidation) cells (fig. $2 \mathrm{~m}$ ) also positive for $\mathrm{NeuN}$ (neurons). A smaller proportion $(<20 \%)$ of hippocampal 4 -HNE-positive cells were also GFAP-positive astrocytes (fig. 2n), but mature (CNPasepositive) oligodendrocytes were not positive for lipid peroxidation (image not shown).

\section{Discussion}

The present study finds that melatonin protects the late gestation fetal brain from the cellular consequences of a severe hypoxic-ischemic insult and the actions of melatonin are likely contributed by several mechanisms. Severe global hypoxia caused by $10 \mathrm{~min}$ of UCO produced fetal hypoxia, hypercapnia, and acidosis, which, in turn, induced lipid peroxidation, brain inflammation, increased the penetration of plasma proteins into brain parenchyma, and initiated astrogliosis. The insult induced apoptotic and/or necrotic cell death in the hippocampus and cerebellum, but not in the thalamus. Maternal melatonin administration before and after UCO abolished neuronal lipid peroxidation, prevented cerebrovascular 

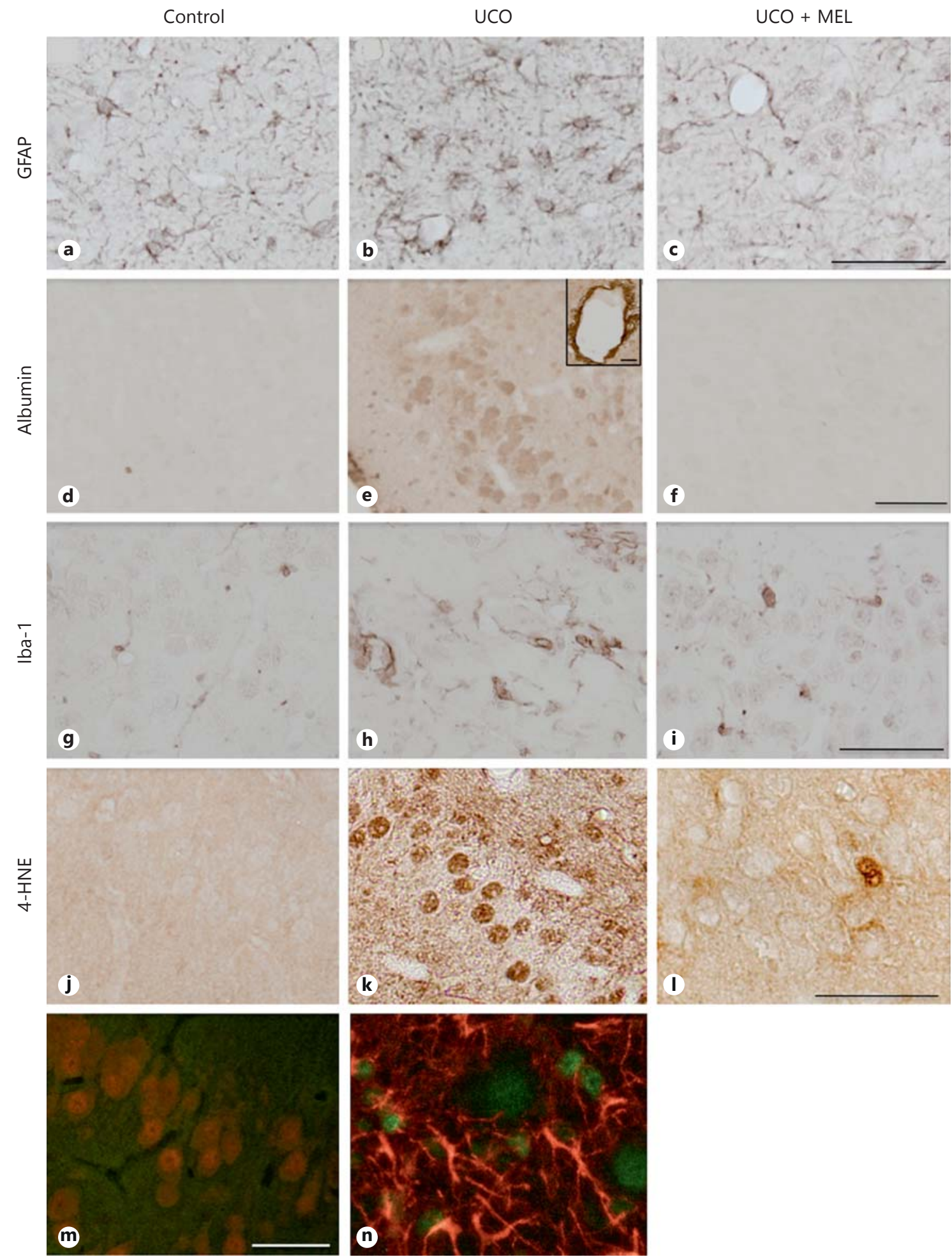

Fig. 2. Photomicrographs showing GFAP (a-c), albumin (d-f), Iba-1 ( $\mathbf{g}-\mathbf{i})$ and $4 \mathrm{HNE}(\mathbf{j}-\mathbf{I})$ immunoreactivity in the CA1 area of the hippocampus in the fetal brain $48 \mathrm{~h}$ after the sham procedure $(\mathbf{a}-\mathbf{j}), \mathrm{UCO}(\mathbf{b}-\mathbf{k})$ and UCO + melatonin (c-I). Scale bars $=50 \mu \mathrm{m}$. $\mathbf{a - c}$ as shown in $\mathbf{c}, \mathbf{d}-\mathbf{f}$ as shown in $\mathbf{f}, \mathbf{g}-\mathbf{i}$ as shown in $\mathbf{i}, \mathbf{j}-\mathbf{I}$ as shown in I. Inset in e shows an albumin-positive blood vessel, scale bar 50 $\mu \mathrm{m}$. Double-label immunohistochemistry in the hippocampus ( $\mathbf{m}$, n) for 4-HNE together with mature neurons (NeuN; $\mathbf{m}$ ) and mature astrocytes (GFAP; $\mathbf{n}$ ) in a UCO brain. Cell types (NeuN and GFAP) were visualized with Alexa Fluor 594 (red) and 4-HNE with Alexa Fluor 488 (green). Scale bar $=50 \mu \mathrm{m}$ as shown in $\mathbf{m}$. 


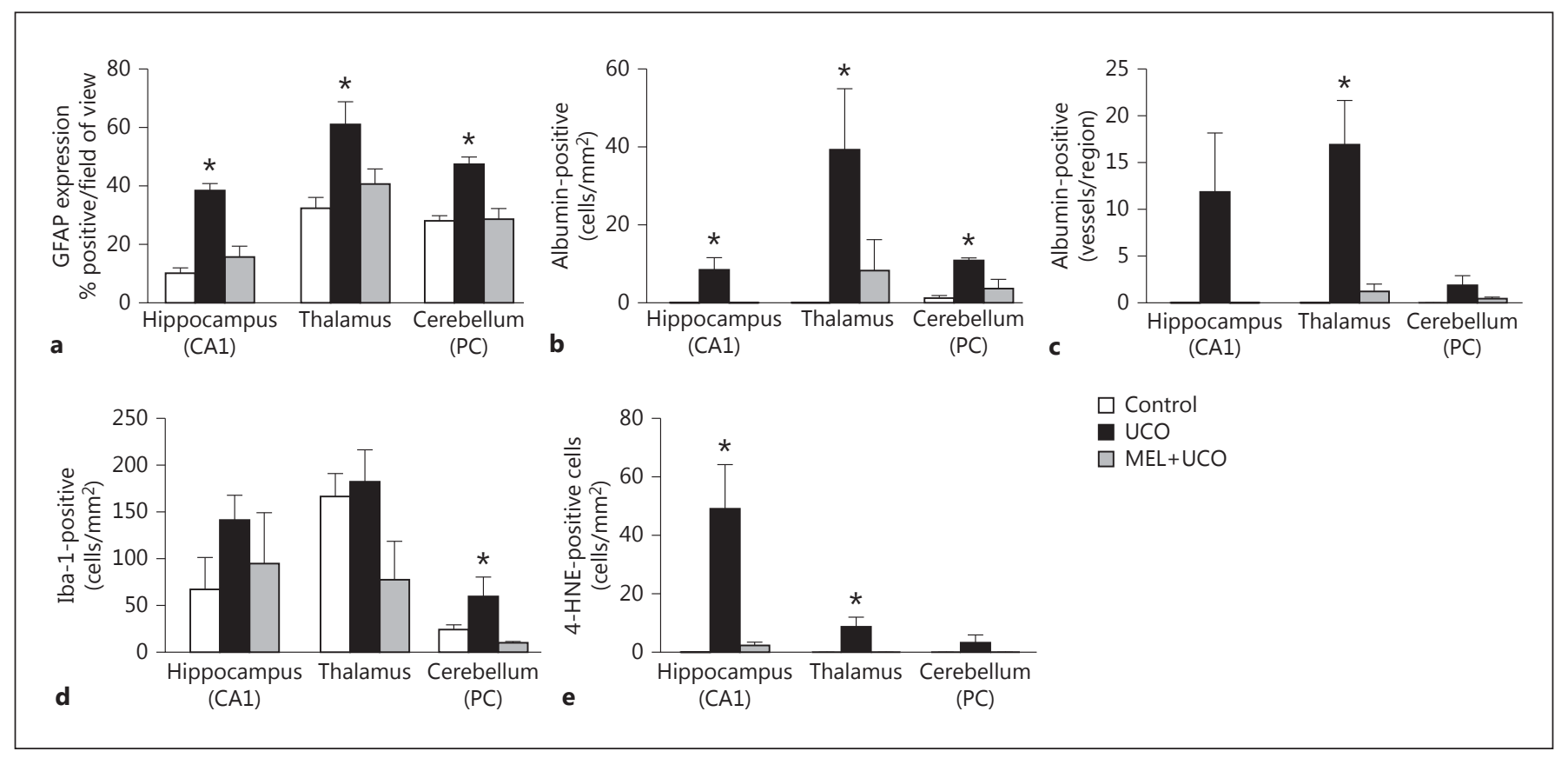

Fig. 3. Expression of GFAP as a percentage of the field of view (a) and the number of positive albumin cells (b), positive albumin vessels (c), positive Iba-1 (d) and 4-HNE (e) cells in the CA1 area of the hippocampus, the thalamus and the cerebellum, in the fetal brain $48 \mathrm{~h}$ after the sham procedure $(\mathrm{n}=5), \mathrm{UCO}(\mathrm{n}=5)$ and UCO + melatonin $(n=5)$. Each bar represents the mean \pm SEM; ${ }^{\star} \mathrm{p}<$ 0.05 , control versus UCO. instability, reduced astrogliosis, reduced inflammation and prevented cell death.

UCO increased lipid peroxidation (4-HNE immunostaining) in the fetal brain, supporting and extending our previous observation of UCO-induced stimulation of hydroxyl radical formation within the brain [5]. In the current study, lipid peroxidation was abolished with maternal melatonin treatment. The overproduction of reactive oxygen species, and particularly the highly toxic hydroxyl radical, is potentially very damaging for tissues, an effect exacerbated in the developing brain with its high lipid content and low endogenous levels of antioxidants [17, 18]. Melatonin is a particularly effective scavenger of the hydroxyl radical $[31,32]$. Oxidative stress is considered a critical mediator of brain injury and functional deficits $[21,33,34]$ and therefore reducing this adverse response following acute hypoxia may protect against cellular damage. This association is supported with our results in the hippocampus, where melatonin ameliorated both cellular lipid peroxidation and pyknosis. However, within the cerebellum we did not find an increase in lipid peroxidation following UCO, but did observe both apoptosis and pyknosis, which were reduced with melatonin administration.

Neuroprotective Melatonin in the Fetal Brain
The hippocampus is vulnerable to acute fetal hypoxia in late-term fetal sheep $[7,35]$ and we also observed a significant increase in the number of pyknotic cells in the CA1 of the hippocampus after UCO. However, at $48 \mathrm{~h}$ after the insult, there was no evidence of increased caspase-3-mediated apoptosis within the hippocampus. Although distinguishing between apoptotic and necrotic cellular degeneration was not the main objective of this study, this result probably reflects the continuum from initial necrosis (pyknosis) through to programmed and delayed apoptosis [36]. The pattern and temporal evolution of neuronal death differs between brain regions, which may explain why we showed both increased apoptosis and pyknosis within the cerebellum after UCO, but did not show significant cellular damage within the thalamus. Thalamic neurons are sensitive to hypoxic insults $[35,37]$, however these cells predominantly respond with programmed apoptotic cell death that is delayed relative to cortical neurons [37] suggesting that the timing of our insult relative to tissue collection may have missed the peak in thalamic neuronal degeneration. Neurons were the principal cell type to stain positively for lipid peroxidation after UCO, reflecting the mature state of the late gestation fetal sheep brain [38], whereas in the preterm 
brain, oligodendrocyte precursor cells are susceptible to oxidative damage contributing to the propensity for preterm white matter damage $[39,40]$. Melatonin also provides white matter protection in the preterm brain, protecting oligodendrocyte maturation in growth-restricted rats [41].

The antioxidant actions of melatonin are complemented by its ability to suppress neuro-inflammatory responses by preventing translocation of nuclear factor- $\kappa B$ and reducing subsequent pro-inflammatory cytokine production [20, 29]. Accordingly, we also assessed the distribution and number of Iba-1-positive microglia cells within the brain. We observed an increase in the number of activated microglia in the cerebellum after the hypoxicischemic insult, but there was no change within the hippocampus or thalamus. The administration of melatonin in UCO animals ameliorated this cerebellar microglial response. There was no evidence of lipid peroxidation within the cerebellum following UCO, and therefore the finding that melatonin mediated the inflammatory microglial cell response within this region is notable, since both apoptotic and necrotic cell death were reduced with melatonin treatment. This finding supports an anti-inflammatory role for melatonin, however it is also known that oxidative stress and inflammation are intimately linked; reactive oxygen species upregulate inflammatory cytokine production via nuclear factor- $\kappa \mathrm{B}$ activation [42] and conversely, activation of brain microglia can induce release of free radicals [43]. The current study cannot separate anti-inflammatory and antioxidant properties of melatonin but, given the strong association between these two systems, it appears that melatonin is a particularly useful neuroprotective agent for the perinatal brain.

Further, we observed that melatonin reduced plasma albumin extravasation in response to acute hypoxia, indicative of cerebrovascular stabilization. The blood-brain barrier (BBB) is relatively impermeable to plasma proteins, such as albumin, in the normal near-term fetal sheep brain, as shown in the current study by the absence of albumin in control brains. Indeed, increased BBB permeability is considered a hallmark of brain injury [44], allowing access of blood-borne products into the extracellular space of the brain. Astrocytes are fundamental in the development and maintenance of the $\mathrm{BBB}$, with endfeet of astocytes ensheathing brain capillaries [45] and inducing expression of tight-junction proteins [44]. Together with providing cerebrovascular stability, maternal melatonin treatment reduced GFAP-positive astrogliosis in the fetal brain after UCO, restoring the normal, nonhypertrophic morphology of astrocytes. BBB breakdown following hypoxic insults has been the subject of review and is most likely contributed by changes in pro-inflammatory cytokines, nitric oxide and vascular endothelial growth factor [44]. As described above, melatonin has anti-inflammatory actions [29] but it also decreases vascular endothelial growth factor and nitric oxide within the brain [46], suggesting that decreased vascular permeability and astrogliosis with melatonin treatment in the current study are likely to be secondary protective benefits.

The present study shows that treating the mother with melatonin can protect the near-term fetal brain against the effects of global perinatal hypoxia. Brain lipid peroxidation was reduced with melatonin, preferentially protecting the neurons, as they were the principal cellular target for oxidative stress. Melatonin was also anti-inflammatory within the cerebellum, reducing the number of activated microglia cells. Astrogliosis and increased BBB permeability were apparent in hypoxic fetuses, and ameliorated with melatonin. Given the strong safety profile of melatonin, we suggest that melatonin should be considered as an effective therapy for use in complicated pregnancies and at birth to protect the perinatal brain when hypoxia-induced brain damage may occur.

\section{Acknowledgements}

The authors wish to acknowledge the technical assistance of Jan Loose and funding provided by the Victorian Government's Operational Infrastructure Support Program. This project was supported by a grant from the National Health and Medical Research Council of Australia.

References

1 Vannucci RC: Experimental biology of cerebral hypoxia-ischemia: relation to perinatal brain damage. Pediatr Res 1990;27:317-326.

2 Dilenge ME, Majnemer A, Shevell MI: Longterm developmental outcome of asphyxiated term neonates. J Child Neurol 2001;16:781792

3 Yager JY: Animal models of hypoxic-ischemic brain damage in the newborn. Semin Pediatr Neurol 2004;11:31-46.

4 Castillo-Melendez M, Chow JA, Walker DW: Lipid peroxidation, caspase- 3 immunoreactivity, and pyknosis in late-gestation fetal sheep brain after umbilical cord occlusion. Pediatr Res 2004;55:864-871.

5 Miller SL, Yan EB, Castillo-Melendez M, Jenkin G, Walker DW: Melatonin provides neuroprotection in the late-gestation fetal sheep brain in response to umbilical cord occlusion. Dev Neurosci 2005;27:200-210. 
6 Gunn AJ, Bennet L: Fetal hypoxia insults and patterns of brain injury: insights from animal models. Clin Perinatol 2009;36:579-593.

7 Mallard EC, Williams CE, Johnston BM, Gluckman PD: Increased vulnerability to neuronal damage after umbilical cord occlusion in fetal sheep with advancing gestation. Am J Obstet Gynecol 1994;170:206-214.

8 Mallard EC, Williams CE, Johnston BM, Gluckman PD: Neuronal damage in the developing brain following intrauterine asphyxia. Reprod Fertil Dev 1995;7:647-653.

-9 Duncan JR, Camm E, Loeliger M, Cock ML, Harding R, Rees SM: Effects of umbilical cord occlusion in late gestation on the ovine fetal brain and retina. J Soc Gynecol Investig 2004; 11:369-376.

10 Volpe JJ: Neurobiology of periventricular leukomalacia in the premature infant. Pediatr Res 2001;50:553-562.

-11 Mallard C, Welin AK, Peebles D, Hagberg H, Kjellmer I: White matter injury following systemic endotoxemia or asphyxia in the fetal sheep. Neurochem Res 2003;28:215-223.

12 Volpe JJ: Perinatal brain injury: from pathogenesis to neuroprotection. Ment Retard Dev Disabil Res Rev 2001;7:56-64.

13 Yawno T, Yan EB, Walker DW, Hirst JJ: Inhibition of neurosteroid synthesis increases asphyxia-induced brain injury in the late gestation fetal sheep. Neuroscience 2007;146: 1726-1733.

14 Ferriero DM: Neonatal brain injury. N Engl J Med 2004;351:1985-1995.

15 Gunn AJ, Battin M, Gluckman PD, Gunn TR, Bennet L: Therapeutic hypothermia: from lab to NICU. J Perinat Med 2005;33:340-346.

16 Yan EB, Unthank JK, Castillo-Melendez M, Miller SL, Langford SJ, Walker DW: Novel method for in vivo hydroxyl radical measurement by microdialysis in fetal sheep brain in utero. J Appl Physiol 2005;98:2304-2310.

-17 Gitto E, Pellegrino S, Gitto P, Barberi I, Reiter RJ: Oxidative stress of the newborn in the preand postnatal period and the clinical utility of melatonin. J Pineal Res 2009;46:128-139.

-18 Saugstad OD: Mechanisms of tissue injury by oxygen radicals: implications for neonatal disease. Acta Paediatr 1996;85:1-4.

-19 Aversa S, Pellegrino S, Barberi I, Reiter RJ, Gitto E: Potential utility of melatonin as an antioxidant during pregnancy and in the perinatal period. J Matern Fetal Neonatal Med 2012;25:207-221.

20 Welin AK, Svedin P, Lapatto R, Sultan B, Hagberg H, Gressens P, Kjellmer I, Mallard C: Melatonin reduces inflammation and cell death in white matter in the mid-gestation fetal sheep following umbilical cord occlusion. Pediatr Res 2007;61:153-158.
21 Miller SL, Wallace EM, Walker DW: Antioxidant therapies: a potential role in perinatal medicine Neuroendocrinology 2012;96:13-23.

22 Fulia F, Gitto E, Cuzzocrea S, Reiter RJ, Dugo L, Gitto P, Barberi S, Cordaro S, Barberi I: Increased levels of malondialdehyde and nitrite/ nitrate in the blood of asphyxiated newborns: reduction by melatonin. J Pineal Res 2001;31: 343-349.

23 Miller SL, Yan E, Castillo-Melendez M, Jenkin G, Walker DW: Melatonin protects against hydroxyl radical induced damage in a fetal sheep model of acute asphyxia. Dev Neurosci 2005;27:200-210.

24 Watanabe K, Wakatsuki A, Shinohara K, Ikenoue N, Yokota K, Fukaya T: Maternally administered melatonin protects against ischemia and reperfusion-induced oxidative mitochondrial damage in premature fetal rat brain. J Pineal Res 2004;37:276-280.

25 Wakatsuki A, Okatani Y, Shinohara K, Ikenoue N, Fukaya T: Melatonin protects against ischemia/reperfusion-induced oxidative damage to mitochondria in fetal rat brain. J Pineal Res 2001;31:167-172.

26 Hamada F, Watanabe K, Wakatsuki A, Nagai R, Shinohara K, Hayashi Y, Imamura R, Fukaya T: Therapeutic effects of maternal melatonin administration on ischemia/reperfusion-induced oxidative cerebral damage in neonatal rats. Neonatology 2010;98:33-40.

27 Koh PO: Melatonin attenuates decrease of protein phosphatase $2 \mathrm{~A}$ subunit $\mathrm{B}$ in ischemic brain injury. J Pineal Res 2012;52:57-61.

28 Richter HG, Hansell JA, Raut S, Giussani DA: Melatonin improves placental efficiency and birth weight and increases the placental expression of antioxidant enzymes in undernourished pregnancy. J Pineal Res 2009;46: 357-364.

29 Esposito E, Cuzzocrea S: Anti-inflammatory activity of melatonin in central nervous system. Curr Neuropharmacol 2010;8:228-242.

30 Yan E, Castillo-Melendez M, Nicholls T, Hirst J, Walker D: Cerebrovascular responses in the fetal sheep brain to low-dose endotoxin. Pediatr Res 2004;55:855-863.

-31 Galano A, Tan DX, Reiter RJ: Melatonin as a natural ally against oxidative stress: a physicochemical examination. J Pineal Res 2011;51: 1-16.

32 Poeggeler B, Saarela S, Reiter RJ, Tan DX, Chen LD, Manchester LC, Barlow-Walden LR: Melatonin - a highly potent endogenous radical scavenger and electron donor: new aspects of the oxidation chemistry of this indole accessed in vitro. Ann NY Acad Sci 1994;738: 419-420.

33 Urabe T, Yamasaki Y, Hattori N, Yoshikawa M, Uchida K, Mizuno Y: Accumulation of 4-hydroxynonenal-modified proteins in hippocampal CA1 pyramidal neurons precedes delayed neuronal damage in the gerbil brain. Neuroscience 2000;100:241-250.
34 Drobyshevsky A, Luo K, Derrick M, Yu L, Du H, Prasad PV, Vasquez-Vivar J, BatinicHaberle I, Tan S: Motor deficits are triggered by reperfusion-reoxygenation injury as diagnosed by MRI and by a mechanism involving oxidants. J Neurosci 2012;32:5500-5509.

35 Castillo-Melendez M, Chow JA, Walker DW: Lipid peroxidation, caspase-3 immunoreactivity, and pyknosis in late-gestation fetal sheep brain after umbilical cord occlusion. Pediatr Res 2004;55:864-871.

- 36 Northington FJ, Zelaya ME, O'Riordan DP, Blomgren K, Flock DL, Hagberg H, Ferriero DM, Martin LJ: Failure to complete apoptosis following neonatal hypoxia-ischemia manifests as 'continuum' phenotype of cell death and occurs with multiple manifestations of mitochondrial dysfunction in rodent forebrain. Neuroscience 2007;149:822-833.

- 37 Northington FJ, Ferriero DM, Martin LJ: Neurodegeneration in the thalamus following neonatal hypoxia-ischemia is programmed cell death. Dev Neurosci 2001;23:186-191.

38 Back SA, Riddle A, Dean J, Hohimer AR: The instrumented fetal sheep as a model of cerebral white matter injury in the premature infant. Neurotherapeutics 2012;9:359-370.

39 Back SA: Perinatal white matter injury: the changing spectrum of pathology and emerging insights into pathogenetic mechanisms. Ment Retard Dev Disabil Res Rev 2006;12:129-140.

40 Back SA, Riddle A, Hohimer AR: Role of instrumented fetal sheep preparations in defining the pathogenesis of human periventricular white-matter injury. J Child Neurol 2006; 21:582-589.

41 Olivier P, Fontaine RH, Loron G, Van Steenwinckel J, Biran V, Massonneau V, Kaindl A, Dalous J, Charriaut-Marlangue C, Aigrot MS, et al: Melatonin promotes oligodendroglial maturation of injured white matter in neonatal rats. PLoS One 2009;4:e7128.

$42 \mathrm{Li}$ N, Karin M: Is NF- $\kappa B$ the sensor of oxidative stress? FASEB J 1999;13:1137-1143.

43 Dringen R: Oxidative and antioxidative potential of brain microglial cells. Antioxid Redox Signal 2005;7:1223-1233.

44 Ballabh P, Braun A, Nedergaard M: The blood-brain barrier: an overview: structure, regulation, and clinical implications. Neurobiol Dis 2004;16:1-13.

45 Abbott NJ: Astrocyte-endothelial interactions and blood-brain barrier permeability. J Anat 2002;200:629-638.

46 Kaur C, Sivakumar V, Lu J, Tang FR, Ling EA: Melatonin attenuates hypoxia-induced ultrastructural changes and increased vascular permeability in the developing hippocampus. Brain Pathol 2008;18:533-547. 